\title{
KARE Genomewide Association Study of Blood Pressure Using Imputed SNPs
}

\author{
Kyung-Won Hong ${ }^{1 \pi}$, Ji-Eun Lim ${ }^{1 \pi}$, Young-Jin \\ $\mathrm{Kim}^{2}$, Nam H. Cho ${ }^{3}$, Chol Shin $^{4}$ and Bermseok \\ $\mathrm{Oh}^{1 *}$
}

${ }^{1}$ Department of Biomedical Engineering, School of Medicine, Kyung Hee University, Seoul 130-701, Korea, ${ }^{2}$ Center for Genome Science, National Institute of Health, Seoul, Korea, ${ }^{3}$ Department of Preventive Medicine, Ajou University School of Medicine, Suwon 443-749, Korea, ${ }^{4}$ Department of Internal Medicine, Korea University Ansan Hospital, Ansan 425-707, Korea

\begin{abstract}
The imputation of untyped SNPs enables researchers to validate association findings across SNP arrays and also enables them to test a large number of SNPs to reveal the fine structure of the association peak, facilitating interpretation of the results and the location of causal polymorphisms. In this study, we applied the imputation method to a genomewide association study and recapitulated the previously associated gene loci of blood pressure traits in Korean cohorts. A total of $1,827,004$ SNPs were imputed by the IMPUTE program, and we conducted a genomewide association study for systolic and diastolic blood pressure. While no SNPs passed the Bonferroni correction $p$-value $\left(p=2.74 \times 10^{-8}\right.$ for $1,827,004$ SNPs), 12 novel loci for systolic blood pressure and 16 novel loci for diastolic blood pressure were detected by imputed SNPs, with $10^{-5}<\mathrm{p}$-value $<10^{-4}$. Moreover, 7 regions (ATP2B1, 10p15.1, ARHGEF12, ALX4, LIPC, $7 q 31.1$, and TCF7L2) out of 14 genetic loci that were previously reported revealed that the imputed SNPs had lower p-values than those of genotyped SNPs. Moreover, a nonsynonymous SNP in the CSMD1 gene, one of the 14 genes, was found to be associated with systolic blood pressure $(p<0.05)$. These results suggest that the imputation method can facilitate the discovery of novel SNPs as well as enhance the fine structure of the association peak in the loci.
\end{abstract}

Keywords: blood pressure, genomewide association study, Korea Association Resource, imputation, single nucleo-

\footnotetext{
"Hong, K.W. and Lim, J.E. contributed equally to this work. ${ }^{*}$ Corresponding author: E-mail ohbs@khu.ac.kr

Tel +82-2-961-0617, Fax +82-2-961-5515

Accepted 24 August 2010
}

tide polymorphism

\section{Introduction}

The human genome contains $\sim 10$ million single nucleotide polymorphisms (SNPS), but only a small fraction of them has been assayed using current high-density microarrays, such as Illumina and Affymetrix platforms. To enhance an original association study, the genotypes of untyped SNPs can be imputed based on nearby markers and can be tested for association with phenotypes of interest. This strategy enables researchers to easily replicate and compare previous findings across array types and enables them to test a large number of SNPs to reveal the fine structure of the association peak, facilitating interpretation of the results and the location of causal polymorphisms (Hao et al., 2009). By using the imputed SNPs, Tobin and colleagues identified a significant association of the WNT1 gene with diastolic blood pressure, and Knouff and colleagues identified a significant association between the CETP gene and diastolic blood pressure (Knouff et al., 2008; Tobin et al., 2008).

The first genomewide association study for large cohorts in Korea was conducted by the Korea National Institute of Health $(\mathrm{KNIH})$ and reported associations with 8 quantitative traits, including blood pressure (Cho et al., 2009). The Korean GWAS suggested a total of 14 loci for blood pressure, 2 (ATP2B1 and 10 p15.1) of which were significant association loci ( $p$-value $<10^{-5}$ ); the remaining 12 (ARHGEF12, CSMD1, ARSG, SGSM1, GJA8, ALX4, 2q31.1, 15q22.1, LIPC, CSK, 7q21.13, 7q31.1, 10q25.2) were suggestive loci $\left(10^{-5}<\mathrm{p}\right.$-value $\left.<10^{-4}\right)$. To enhance the original association study, a total of $1,827,004$ imputed SNPs were reanalyzed for their association with blood pressure in this study.

\section{Methods}

Subjects and their genotypes were reported in a previous genomewide association study (Cho et al., 2009). Briefly, subjects came from 2 community-based cohorts, the rural community Ansung and the urban community Ansan, in KyungGi-Do province, near Seoul, Korea. Most DNA samples were isolated from the peripheral blood of participants and genotyped using the Affymetrix Genomewide Human SNP array 5.0 (Affymetrix, Inc., Santa Clara, CA, USA). After the quality control steps, we finally used 
7751 individuals for blood pressure traits. The 351,677 genotyped SNPs had a missing gene call rate below 0.1 , a minor allele frequency (MAF) higher than 0.01 , and no deviation from Hardy-Weinberg Equilibrium (HWE) $\left(p>1 \times 10^{-6}\right)$ (WTCCC, 2007).

Table 1. Basic characteristics of study subjects

\begin{tabular}{lc}
\hline \multicolumn{1}{c}{ Variables } & $\begin{array}{c}\text { Count/ } \\
\text { mean } \pm \text { standard deviation }\end{array}$ \\
\hline Number of individuals & 7,751 \\
Gender [men (\%)/women (\%)] & $3,747(50) / 3,804(50)$ \\
Age & $51.44 \pm 8.79$ \\
BMI & $24.4 \pm 3.2$ \\
SBP & $115.65 \pm 17.25$ \\
DBP & $74.21 \pm 11.27$ \\
\hline
\end{tabular}

The basic characteristics and blood pressures of the subjects are listed in Table 1. For this study, blood pressure measurements were taken 3 times in the supine position. Before the first measurement, participants rested for 5 minutes, and 3 measurements were made at least 30 seconds apart. The average of the 3 measurements was used for this study.

The KARE dataset, comprising 351,677 SNPs for 7751 individuals, was merged with that of International HapMap Phase II JPT (Japanese)+HCB (Chinese) panel 2 . The genotypes of the KARE individuals were imputed using IMPUTE (Howie et al., 2009).

Most statistical analyses were performed using PLINK, version 1.07 (http://pngu.mgh.harvard.edu/ purcell/plink/) (Purcell et al., 2007) and SAS (version 9.1; SAS Institute Inc., Cary, NC, USA). Systolic blood pressure (SBP) and diastolic blood pressure (DBP) were tested for their as-

Table 2. Novel SNP loci identified by the imputed SNPS

\begin{tabular}{|c|c|c|c|c|}
\hline \multirow{2}{*}{ Locus } & \multirow{2}{*}{ Nearby genes } & \multirow{2}{*}{ No. of $\mathrm{SNPs}^{\mathrm{a}}$} & \multicolumn{2}{|c|}{$p$-value's range } \\
\hline & & & Min. & Max. \\
\hline \multicolumn{5}{|c|}{ Systolic blood pressure } \\
\hline $1 \mathrm{q} 25.1$ & CACYBP/MRPS14/TNN/KIAA0040/TNR & 1 & $9.67 \times 10^{-5}$ & \\
\hline TNR & & 1 & $9.90 \times 10^{-5}$ & \\
\hline ALK & & 1 & $5.29 \times 10^{-5}$ & \\
\hline $2 q 14.2$ & TMEM185B/RALB/INHBB/LOC84931 & 1 & $8.35 \times 10^{-5}$ & \\
\hline $3 p 24.3$ & ZNF385D & 1 & $2.06 \times 10^{-5}$ & \\
\hline $3 q 25.1$ & HPS3/CP/TM4SF18/TM4SF1/TM4SF4/WWTR1 & 1 & $2.83 \times 10^{-5}$ & \\
\hline SLIT2 & & 2 & $8.30 \times 10^{-5}$ & $9.40 \times 10^{-5}$ \\
\hline $4 q 25$ & PAPSS1 & 1 & $6.78 \times 10^{-5}$ & \\
\hline $4 q 32.3$ & NPY1R/NPY5R/TKTL2/C4orf43/MARCH1 & 1 & $7.55 \times 10^{-5}$ & \\
\hline EBF1 & & 2 & $7.69 \times 10^{-5}$ & $9.90 \times 10^{-5}$ \\
\hline SEMA3E & & 1 & $9.76 \times 10^{-5}$ & \\
\hline $8 p 12$ & NRG1 & 1 & $7.55 \times 10^{-5}$ & \\
\hline \multicolumn{5}{|c|}{ Diastolic blood pressure } \\
\hline $14 \mathrm{q} 23.2$ & HIF1A/SNAPC1/SYT16/FLJ43390 & 1 & $8.84 \times 10^{-5}$ & \\
\hline $17 q 25.3$ & SEPT9 & 2 & $2.36 \times 10^{-5}$ & $2.48 \times 10^{-5}$ \\
\hline $18 q 21.33$ & $\mathrm{CDH} 20 / \mathrm{RNF} 152$ & 1 & $7.27 \times 10^{-5}$ & \\
\hline $20 \mathrm{q} 13.13$ & SULF2 & 1 & $9.32 \times 10^{-5}$ & \\
\hline $2 q 33.1$ & SPATS2L/KCTD18 & 7 & $3.35 \times 10^{-5}$ & $8.76 \times 10^{-5}$ \\
\hline $2 \mathrm{q} 37.1$ & $\begin{array}{l}\text { UGT1A1/3/4/5/6/7/8/9/10/DNAJB3/HJURP/MSL3L2/TRP } \\
\text { M8/SPP2 }\end{array}$ & 6 & $6.07 \times 10^{-5}$ & $9.70 \times 10^{-5}$ \\
\hline $3 p 12.3$ & $\mathrm{ROBO} 1$ & 1 & $8.07 \times 10^{-5}$ & \\
\hline $3 p 24.3$ & ZNF385D & 1 & $8.26 \times 10^{-5}$ & \\
\hline $5 q 12.1$ & PDE4D/PART1/DEPDC1B/ELOVL7/ERCC8/NDUFAF2 & 1 & $3.70 \times 10^{-5}$ & \\
\hline $8 p 22$ & SGCZ/TUSC3 & 1 & $1.24 \times 10^{-5}$ & \\
\hline $8 p 23.1$ & TDH/C8orf12/FAM167A/BLK/GATA4/NEIL2/FDFT1/CTSB & 1 & $7.19 \times 10^{-5}$ & \\
\hline $\mathrm{BCR}$ & & 2 & $3.62 \times 10^{-5}$ & $4.58 \times 10^{-5}$ \\
\hline ELOVL7 & & 1 & $1.91 \times 10^{-5}$ & \\
\hline LOC100188947 & & 1 & $5.29 \times 10^{-5}$ & \\
\hline PTPRT & & 1 & $2.48 \times 10^{-5}$ & \\
\hline TRPM8 & & 2 & $1.74 \times 10^{-5}$ & $2.27 \times 10^{-5}$ \\
\hline
\end{tabular}

Boldface highlightsthe locus associated with both systolic and diastolic blood pressure, ${ }^{a}$ Number of significant imputed SNPs in the locus. 
Table 3. Comparison of the association results of genotyped and imputed SNPs in the reported gene regions

\begin{tabular}{|c|c|c|c|c|c|c|c|c|c|c|c|}
\hline \multicolumn{3}{|c|}{ Candidate region } & \multicolumn{3}{|c|}{ Number of SNPs } & \multicolumn{4}{|c|}{ Top SNPs } & \multicolumn{2}{|c|}{ LD } \\
\hline Cho et al., Table 1 & Locus & Franking & Total & Genotypec & Imputed & Reported & $p$-value & Imputed & $p$-value & $r^{2}$ & $D^{\prime}$ \\
\hline \multicolumn{12}{|l|}{ Systolic blood pressure } \\
\hline ATP2B1 & $12 q 21.33$ & $\pm 20 \mathrm{~kb}$ & 29 & 14 & 15 & rs17249754 & $3.4 \times 10^{-7}$ & rs12579302 & $2.4 \times 10^{-7}$ & 1.0 & 1.0 \\
\hline rs715987 & 10p15.1 & $\pm 500 \mathrm{~kb}$ & 967 & 284 & 683 & rs715987 & $1.1 \times 10^{-5}$ & rs10795186 & $9.3 \times 10^{-6}$ & 1.0 & 1.0 \\
\hline \multicolumn{12}{|l|}{ Diastolic blood pressure } \\
\hline ATP2B1 & $12 q 21.33$ & $\pm 20 \mathrm{~kb}$ & 29 & 14 & 15 & rs17249754 & $3.8 \times 10^{-7}$ & rs12579302 & $4.7 \times 10^{-7}$ & 1.0 & 1.0 \\
\hline \multicolumn{12}{|c|}{ Cho et al., Supplementary Table 6} \\
\hline \multicolumn{12}{|l|}{ Systolic blood pressure } \\
\hline ARHGEF12 & $11 q 23.3$ & $\pm 20 \mathrm{~kb}$ & 81 & 33 & 48 & rs10790381 & $5.4 \times 10^{-6}$ & rs17123861 & $4.9 \times 10^{-6}$ & 1.0 & 1.0 \\
\hline CSMD1 & $8 \mathrm{p} 23.2$ & $\pm 20 \mathrm{~kb}$ & 3,335 & 989 & 2,346 & rs995322 & $1.3 \times 10^{-5}$ & rs6558796 & $2.5 \times 10^{-5}$ & 1.0 & 1.0 \\
\hline ARSG & $17 q 24.2$ & $\pm 20 \mathrm{~kb}$ & 81 & 41 & 40 & rs12945290 & $3.8 \times 10^{-5}$ & rs12451531 & $4.2 \times 10^{-5}$ & 0.9 & 1.0 \\
\hline SGSM1 & $22 q 11.23$ & $\pm 20 \mathrm{~kb}$ & 174 & 31 & 143 & rs7287595 & $4.4 \times 10^{-5}$ & rs5760712 & $3.3 \times 10^{-2}$ & 0.0 & 0.1 \\
\hline GJA8 & $1 \mathrm{q} 21.1$ & $\pm 20 \mathrm{~kb}$ & 12 & 5 & 7 & rs7544630 & $3.1 \times 10^{-5}$ & rs12028407 & $4.0 \times 10^{-2}$ & 0.0 & 0.3 \\
\hline ALX4 & $11 \mathrm{p} 11.2$ & $\pm 20 \mathrm{~kb}$ & 60 & 23 & 37 & rs879238 & $1.7 \times 10^{-4}$ & rs7481493 & $1.0 \times 10^{-5}$ & 0.8 & 1.0 \\
\hline \multicolumn{12}{|l|}{ Diastolic blood pressure } \\
\hline rs1006815 & $2 \mathrm{q} 31.1$ & $\pm 500 \mathrm{~kb}$ & 652 & 208 & 444 & rs1006815 & $2.0 \times 10^{-4}$ & rs1371441 & $2.4 \times 10^{-4}$ & 1.0 & 1.0 \\
\hline LIPC & $15 q 22.1$ & $\pm 20 \mathrm{~kb}$ & 145 & 73 & 72 & rs11631342 & $3.4 \times 10^{-5}$ & rs11636642 & $2.8 \times 10^{-5}$ & 1.0 & 1.0 \\
\hline CSK & $15 q 24.1$ & $\pm 20 \mathrm{~kb}$ & 39 & 8 & 31 & rs1378942 & $4.6 \times 10^{-5}$ & rs4886410 & $7.2 \times 10^{-5}$ & 1.0 & 1.0 \\
\hline rs2564438 & $7 q 21.13$ & $\pm 500 \mathrm{~kb}$ & 618 & 156 & 462 & rs2564438 & $7.1 \times 10^{-5}$ & rs4577890 & $1.3 \times 10^{-4}$ & 0.5 & 1.0 \\
\hline rs2940371 & $7 \mathrm{q} 31.1$ & $\pm 500 \mathrm{~kb}$ & 729 & 169 & 560 & rs2940371 & $2.1 \times 10^{-4}$ & rs7799583 & $1.1 \times 10^{-4}$ & 0.9 & 1.0 \\
\hline TCF7L2 & $10 q 25.2$ & $\pm 20 \mathrm{~kb}$ & 76 & 38 & 38 & rs10787472 & $3.6 \times 10^{-5}$ & rs10885405 & $9.6 \times 10^{-6}$ & 0.4 & 1.0 \\
\hline
\end{tabular}

Top SNPs: SNPs that have the lowest p-value in the region, as evaluated by linear regression analysis, controlling for area, age, sex, and BMI LD: linkage disequilibrium between reported and imputed Top SNPs.

sociation by linear regression analysis with an additive model (1-d.f.) after adjustments for area, age, sex, and BMI.

\section{Results and Discussion}

Using a filtering scheme of INFO $\geq 0.5$ and posterior probability $\geq 0.9$, a total of $1,827,004$ SNPs were imputed and analyzed for the genomewide association study of blood pressure traits. While no SNP passed the Bonferroni correction $p$-value $\left(p=2.74 \times 10^{-8}\right.$ for 1,827,004 SNPs), 12 novel loci for systolic blood pressure (SBP) and 16 loci for diastolic blood pressure (DBP) were detected by the imputed SNPs with $10^{-5}$ $<$ p-value $<10^{-4}$ (Table 2). Most of the loci had lower than 2 SNPs of significance near the region, except 20q13.13 and 2q33.1, which had 7 and 6 SNPs of significance, respectively. Notably, the SNP (rs1027345) near the ZNF385D gene in the $3 p 24.3$ region was significant for both SBP and DBP $\left(p=2.06 \times 10^{-5}\right.$ and $8.26 \times 10^{-5}$, respectively).

In order to compare the signal $p$-values of the imputed and typed SNPs, SNPs that were located in the 14 previously reported genetic loci in the KARE study were investigated (Table 3). A total of 7483 SNPs were extracted from the 1,827,004 imputed SNPs and analyzed for blood pressure. The most significant SNPs and

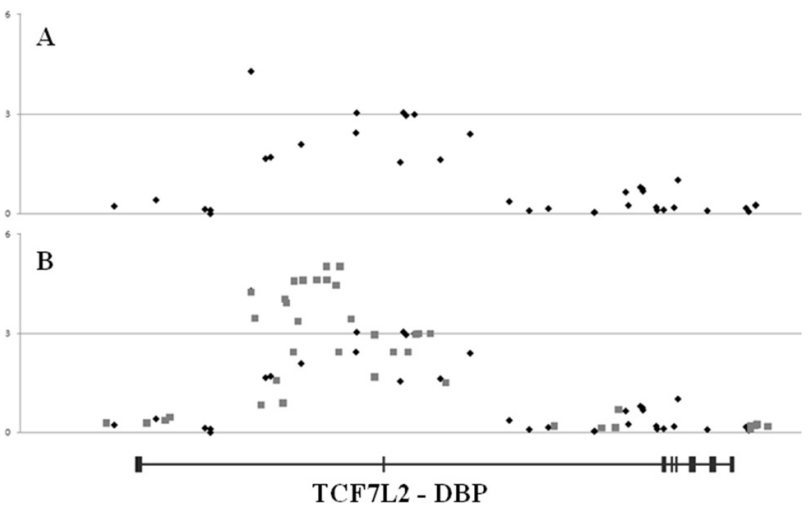

Fig. 1. Signal $(-\log P)$ plots of the association between SNPs of the TCF7L2 region and diastolic blood pressure (DBP). A. genotyped SNPs. B. Both genotyped and imputed SNPs.

their gene names are described in Table 3 . For the extraction, the candidate regions were defined as $\pm 20 \mathrm{~kb}$ for the gene region and $\pm 500 \mathrm{~kb}$ for the intergenic region. Among the 14 loci, 7 loci (ATP2B1, 10p15.1, ARHGEF12, ALX4, LIPC, 7q31.1, and TCF7L2) showed enhanced association with blood pressure by the imputed SNPs.

Imputation methods cover the candidate gene region 
Table 4. Nonsynonymous SNPs among the imputed SNPs and the association results

\begin{tabular}{|c|c|c|c|c|c|c|}
\hline \multirow{2}{*}{ Gene } & \multirow{2}{*}{ SNP } & \multirow{2}{*}{ Protein Change } & \multicolumn{2}{|c|}{ Systolic blood pressure } & \multicolumn{2}{|c|}{ Diastolic blood pressure } \\
\hline & & & $\beta \pm$ s.e. & $\mathrm{p}$ & $\beta \pm$ s.e. & $\mathrm{p}$ \\
\hline CSMD1 & rs11984691 & S3207N & $2.687 \pm 1.105$ & 0.015 & $0.847 \pm 0.749$ & 0.259 \\
\hline ALX4 & rs3824915 & R35T & $0.007 \pm 0.281$ & 0.980 & $0.147 \pm 0.192$ & 0.442 \\
\hline CSK & rs3803568 & R105Q & $0.506 \pm 0.331$ & 0.126 & $0.319 \pm 0.224$ & 0.154 \\
\hline LIPC & rs3829462 & F356L & $0.104 \pm 0.458$ & 0.821 & $-0.125 \pm 0.310$ & 0.687 \\
\hline ARSG & rs3213690 & N30D & $0.201 \pm 0.309$ & 0.515 & $0.279 \pm 0.209$ & 0.184 \\
\hline SGSM1 & rs2073201 & R873K & $-0.172 \pm 0.308$ & 0.576 & $-0.326 \pm 0.208$ & 0.118 \\
\hline
\end{tabular}

densely, which enables one to test a large number of SNPs to reveal the fine structure of the association peak. The association results of the TCF7L2 gene could fall under this case (Fig. 1). While only one SNP with a $\mathrm{p}$-value lower than $10^{-4}$ was present in the peak of the association by the genotyped SNPs, the imputed SNPs showed multiple SNPs ( $p$-value $<10^{-4}$ ) that were associated around the peak, indicating that the imputation methods enhance the fine structure of the GWAS results. Moreover, 6 nonsynonymous SNPs in the 14 genes were identified from the imputed SNPs, and the rs11984691 of CSMD1 revealed an association signal with systolic pressure ( $p$-value=0.015) (Table 4). CSMD1 (CUB and Sushi multiple domains 1 ) is located on chromosome 8p23.2 and is known as a putative suppressor of squamous cell carcinomas of the head and neck (Sun et al., 2001). The gene encodes a 389-KDa transmembrane protein, named for its repeat CUB and sushi domains. Both CUB and sushi domains are found in many other proteins and are thought to be sites of either protein-protein or protein-ligand interactions (Kristiansen et al., 1999; Lau and Scholnick, 2003). The association between the CSMD1 gene and blood pressure was replicated in our previous report (Hong et al., 2010), and the nonsynonymous rs 11984691 might be a causative variation of the CSMD1 gene for the regulation of blood pressure, which waits for its functional analysis in the future.

In conclusion, a total of 1,827,004 SNPs were imputed based on International HapMap Asian data, and a genomewide association study of KARE for systolic and diastolic blood pressure was performed. Even though no SNPs were found to be statistically significant over the Bonferroni corrected p-value, 12 novel loci for systolic blood pressure and 16 novel loci for diastolic blood pressure were identified in the range of $10^{-5}<\mathrm{p}$-value $<10^{-4}$. Additionally, the imputed SNPs revealed better $p$-values in 7 out of 14 genetic loci that were previously reported by the genotyped SNPs. These results suggest that the imputation method can facilitate the discovery of associated SNPs as well as enhance the fine structure of the association in the loci.

\section{Acknowledgments}

The Consortium for Large Scale Genome Wide Association Study was supported by genotyping data (Genome Wide association analysis of community based cohort study; 2007) from the Korean Genome Analysis Project (4845-301) and the Korea National Institute of Health (Korea Center for Disease Control, Ministry for Health, Welfare and Family Affairs), Republic of Korea.

\section{References}

Cho, Y.S., Go, M.J., Kim, Y.J., Heo, J.Y., Oh, J.H., Ban, H.J., Yoon, D., Lee, M.H., Kim, D.J., Park, M., Cha, S.H., Kim, J.W., Han, B.G., Min, H., Ahn, Y., Park, M.S., Han, H.R., Jang, H.Y., Cho, E.Y., Lee, J.E., Cho, N.H., Shin, C., Park, T., Park, J.W., Lee, J.K., Cardon, L., Clarke, G., McCarthy, M.I., Lee, J.Y., Oh, B., and Kim, H.L. (2009). A large-scale genome-wide association study of Asian populations uncovers genetic factors influencing eight quantitative traits. Nat. Genet. 41, 527-534.

Hao, K., Chudin, E., McElwee, J., and Schadt, E.E. (2009). Accuracy of genome-wide imputation of untyped markers and impacts on statistical power for association studies. BMC Genet. 10, 27.

Hong, K.W., Go, M.J., Jin, H.S., Lim, J.E., Lee, J.Y., Han, B.G., Hwang, S.Y., Lee, S.H., Park, H.K., Cho, Y.S., and Oh, B. (2010). Genetic variations in ATP2B1, CSK, ARSG and CSMD1 loci are related to blood pressure and/or hypertension in two Korean cohorts. J. Hum. Hypertens. 24, 367-372.

Howie, B.N., Donnelly, P., and Marchini, J. (2009). A flexible and accurate genotype imputation method for the next generation of genome-wide association studies. PLOS Genet. 5, e1000529.

Knouff, C.W., Lim, N., Song, K., Yuan, X., Walker, M.C., Townsend, R., Waeber, G., Matthews, P.M., Vollenweider, P., Waterworth, D.M., and Mooser, V. (2008). Pharmacological effects of lipid-lowering drugs recapitulate with a larger amplitude the phenotypic effects of common variants within their target genes. Pharmacogenet Genomics 18, 1051-1057.

Kristiansen, M., Kozyraki, R., Jacobsen, C., Nexo, E., Verroust, P.J., and Moestrup, S.K. (1999). Molecular dissection of the intrinsic factor-vitamin B12 receptor, cubi- 
lin, discloses regions important for membrane association and ligand binding. J. Biol. Chem. 274, 20540-20544.

Lau, W.L., and Scholnick, S.B. (2003). Identification of two new members of the CSMD gene family small star, filled. Genomics 82, 412-415.

Purcell, S., Neale, B., Todd-Brown, K., Thomas, L., Ferreira, M.A., Bender, D., Maller, J., Sklar, P., de Bakker, P.I., Daly, M.J., and Sham, P.C. (2007). PLINK: a tool set for whole-genome association and population-based linkage analyses. Am. J. Hum. Genet. 81, 559-575.

Sun, P.C., Uppaluri, R., Schmidt, A.P., Pashia, M.E., Quant, E.C., Sunwoo, J.B., Gollin, S.M., and Scholnick, S.B.
(2001). Transcript map of the $8 p 23$ putative tumor suppressor region. Genomics 75, 17-25.

Tobin, M.D., Timpson, N.J., Wain, L.V., Ring, S., Jones, L.R., Emmett, P.M., Palmer, T.M., Ness, A.R., Samani, N.J., Smith, G.D., and Burton, P.R. (2008). Common variation in the WNK1 gene and blood pressure in childhood: the Avon Longitudinal Study of Parents and Children. Hypertension 52, 974-979.

WTCCC. (2007). Genome-wide association study of 14,000 cases of seven common diseases and 3,000 shared controls. Nature 447, 661-78. 